\title{
Oscillatory fracture paths in thin elastic sheets
}

\author{
Benoit Roman, Pedro M. Reis, Basile Audoly, Simon De Villiers, Vincent Viguié, Denis Vallet \\ PMMH, UMR 7636 CNRS/ESPCI, 10 rue Vauquelin 75231 cedex 5 Paris, France \\ Manchester Center for Nonlinear Dynamics, Dept. physics and astronomy, University of Manchester, M139PL UK \\ LMM, UMR 7607 CNRS/UPMC, 4 place Jussieu, case 162, 75252 Paris cedex 05, France
}

\begin{abstract}
We report a novel mode of oscillatory crack propagation when a cutting tip is driven through a thin brittle polymer film. Experiments show that the amplitude and wavelength of the oscillatory crack paths scale linearly with the width of the cutting tip over a wide range of length scales but are independent of the width of the sheet and of the cutting speed. A simple geometric model is presented, which provides a simple but thorough interpretation of the oscillations. http://www.lmm.jussieu.fr/platefracture/
\end{abstract}

Despite a long history of research into the field of fracture, many puzzling issues remain unsolved. An interesting problem concerns the direction of propagation of the crack tip: when a glass breaks, can the shape of the resulting pieces be predicted? Recent well controlled experiments have yield a variety of interesting behaviours that are a challenge to existing theoretical formulations. An oscillatory instability in dynamic cracks was recently observed in a pre-tensioned thin rubber sheet [6] whose mechanism is still unclear. Another example is the controllable quasi-static propagation of oscillatory cracks in a thin strip of glass submitted to a thermal field [1, 2, 5] which, despite its apparent simplicity, has been stimulating much theoretical study [3, 4].

Here we report novel results on oscillatory fracture paths in a new experimental context: an object, which we denote by cutting tip, is perpendicularly driven through a thin polymer sheet held along its lateral boundaries, and progressively cuts the material as it advances. For large enough cutting tip widths, the crack follows a well defined and highly reproducible oscillatory path that spans a wide range of length scales (Figure 11). In fact, even doing the experiment by hand yields surprisingly regular patterns. The experimental observations of oscillatory motion in this new geometry present a challenge, from a fundamental point of view, to our understanding of crack propagation in thin sheets. Moreover, these ideas should have practical applications since the precise cutting of brittle thin sheets is common in industrial manufacturing.

We perform well controlled experiments with thin sheets of different polymeric materials in a range of thicknesses and investigate the dependence of the resulting fracture paths on the width of the cutting tip. We also propose a simple model that correctly reproduces the behaviour of the fracture and illustrates the geometrical origin of the oscillation.

\section{Experimental oscillatory fracture paths}

A schematic diagram of the apparatus is presented in Figure 2 It consists of a thin flat sheet (dimensions ranging from $6 \times 60 \mathrm{~mm}$ to $12 \times 50 \mathrm{~cm}$ ) clamped along its lateral boundaries and mounted on on a linear translation stage. This stage was driven at constant speed $v$ towards a fixed object, the cutting tip, which could have either rectangular or cylindrical profile with a variety of widths $(0.05 \mathrm{~mm}<w<60 \mathrm{~mm})$. A camera was mounted directly above the apparatus such that the propagating crack was imaged in the cutting tip's frame of reference.

The sheet was initially prepared with a notch on one of its side boundaries to position and initiate the crack. Both bi-oriented polypropylene and cellulose acetate thin sheets were investigated, with thicknesses ranging between 25 and $130 \mu \mathrm{m}$. The sheet's Young's modulus and fracture energy were measured to lie within the ranges $E=1-2 \mathrm{GPa}$ and $\gamma=2-5 \mathrm{~kJ} / \mathrm{m}^{2}$, respectively. Although they are made of polymers, these materials are brittle as they were severely

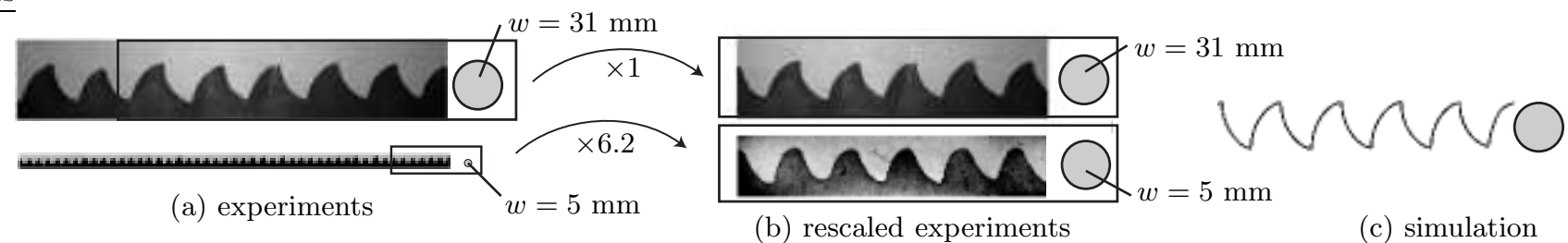

FIG. 1: Right: edge of the sheet seen from above (polypropylene $27 \mu \mathrm{m}$ thick) for two diameters of the cylindrical cutting tip: $w=31 \mathrm{~mm}$ and $5 \mathrm{~mm}$. (a) Raw experimental snapshots of the cracked film edge and (b) after rescaling according to indenter radius; (c) numerical simulation. 

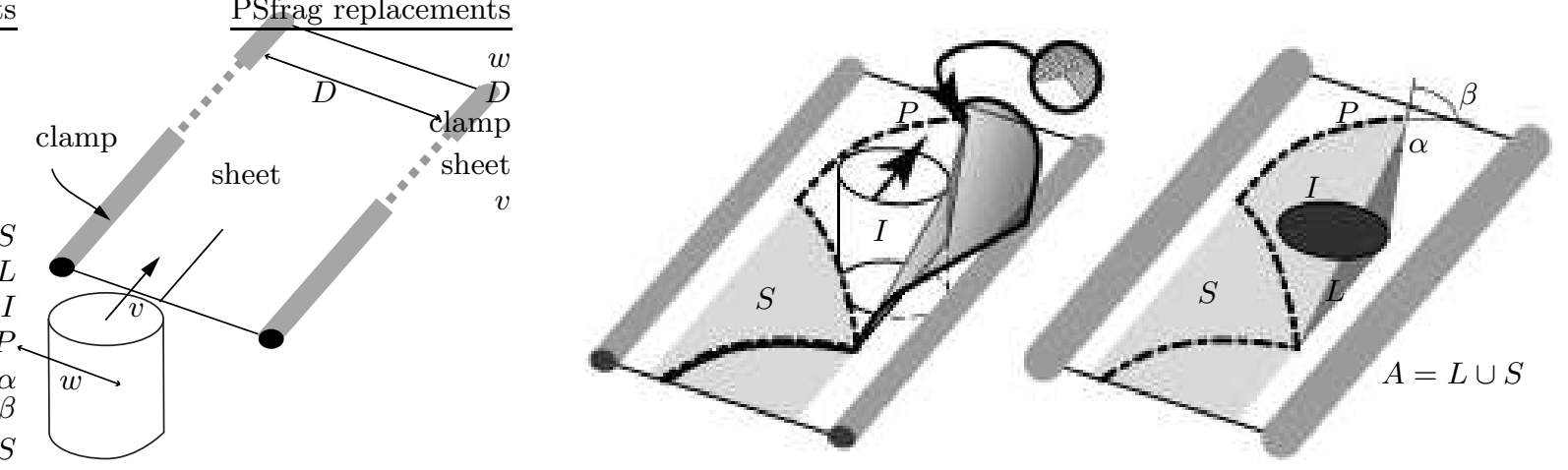

FIG. 2: A cylindrical cutting tip is forced into a clamped sheet with a notch. The cutting tip is displaced at constant speed $v$ with respect to the plate, leading to an oscillatory crack path. Initial configuration of experimental setup (left), typical configuration during fracture (center) and geometrical 2D model (right). The strip is flexible in the smallest convex $S$ (light grey region) containing the crack path $P$, where it can easily bend without stretching. Penetration of the cutting tip beyond this region induces tensiles stresses in the plate. Our simplified 2D geometrical model takes into acount this important feature through the angle $\alpha$ at the tip of the active zone $L$ shown in dark grey: propagation is set to take place in mode $I I I$ loading (inset) along a direction given by $\beta$ when $\alpha$ exceeds a critical value $\alpha_{\mathrm{c}}$.

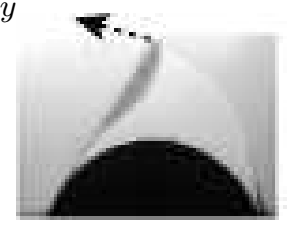

(a)

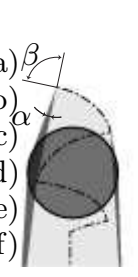

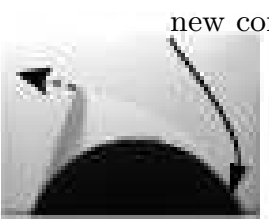

(b)

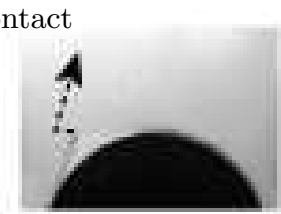

(c)

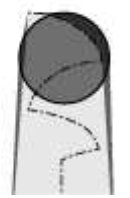

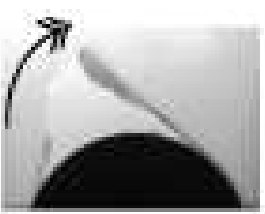

(d)

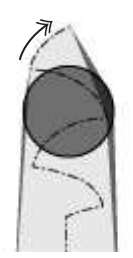

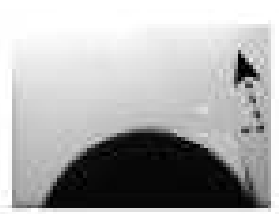

(e)

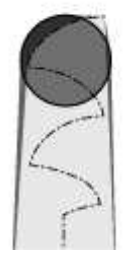

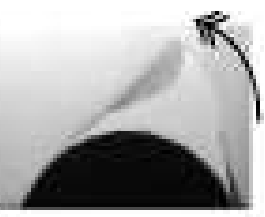

(f)

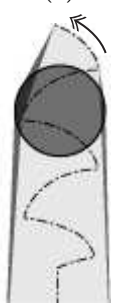

FIG. 3: Similar sequential processes of the crack propagation over a single period (top: experimental cutting tip in black; bottom: corresponding frames in simulation with $\alpha=.153$ and $\beta=\pi / 2$, crack path in dashed line). Frames (a-c): the left hand side of the crack edge is active and the crack tip advances leftwards. In between (a) and (b), the cutting tip crosses the upper part of the crack curve and a new contact appears. A kink then takes place, followed by a dynamical propagation (double arrow), both in the experiments and in the simulation, up to (d) which is the mirror image of the initial frame (a). The second half-period up to (f) is similar. In the simulation, the kink occurs because the active zone (dark grey area) is made up of two components, which rest on either side of the indenter. Change of crack direction between (c) and (d) occurs when the angle $\alpha$ corresponding to the right hand component of the active zone becomes larger than that of the left hand side. Movies available at http://www.lmm.jussieu.fr/platefracture

stretched when processed into thin sheets. As a result, they undergo minimal plastic deformation during fracture propagation but, being thin, can sustain large bending without crack initiation. This explains why they are widely used in the packaging industry (resistant but easy to tear once a notch is started). The oscillatory paths discussed below were not observed in ductile materials.

As the thin sheet is forced through the fixed tip, the material is cut, leaving behind a well defined and highly reproducible oscillatory fracture pattern, some examples of which are shown in Figure 11 As one would expect, for thin enough objects the path is straight. The study of the transition from straight to oscillatory paths will be presented elsewhere [8] and here we focus on the oscillatory regime for large cutting tips, that is well above threshold.

Although these oscillatory patterns are reminiscent of those observed in thermal quenching experiments [1, 2, 5], an important difference is that the oscillation mechanism here arises from a coupling with the large out-of-plane deflection of the thin sheet, as seen in the experimental frames in Figure 3 . 


\section{A simple geometrical model}

We believe that the origin of the oscillations in our experiment lies in the mechanical properties of thin elastic sheets, and in their connection with the geometry of surfaces. Under external constraints, thin elastic sheets bend in order to avoid stretching, since bending energy is small compared to stretching energy [7]. In order to conserve their in-plane lengths and remain unstretched, thin sheets tend to adopt developable shapes, that are the reunion of straight lines, generatrices. Because the lateral edges of the plate are held, these generatrices may not cross the clamped boundaries of the plate and can only end up on the crack path. We thus define a "soft" zone in the plane of the elastic plate by considering the reunion of all the segments ending on the crack path. This is called the convex hull $S$ of the crack path (see Fig. 2 right). In $S$, the sheet can deform by pure bending and can therefore easily accomodate the presence of the cutting tip. However, when the cutting tip penetrates beyond $S$, in a region which we call "hard", the sheet undergoes stretching which eventually leads to crack propagation.

In order to determine the direction of propagation in our thin sheets we have performed a complementary ripping (mode III) experiment by systematically curving and pulling one side of an initial notch. Crack propagation was observed to always follow a well defined and highly reproducible angle $\beta$ with respect to the generatrix of the fold. This angle $\beta$ depends on the material and, since these materials are anisotropic, on the orientation of the ripping, but we consistently measured an angle close to $\pi / 2$ to within $20 \%$. So, to first approximation, we take $\beta=\pi / 2$.

The propagation criterion in our model is the following. We consider the "large" hull $A$, defined as the convex hull of the crack tip plus the horizontal section of cutting object (black disk in the Fig. 2c) altogether. The so called active area $L$ (in dark grey) computed as the set difference $L=A \backslash S$ of the large and the soft hull is the region of the hard zone where penetration of the cutting tip induces tensile stresses. When this "active" area includes a cone with angle $\alpha>\alpha_{\text {c }}$ at the crack tip greater than a critical value (see Fig. 2. $)$, the crack is set to propagate instantaneously in the direction given by the angle $\beta$, until $\alpha$ decreases back to $\alpha_{\mathrm{c}}$ (note that $\alpha$ indeed decreases during crack advance since the soft zone expands). The angle $\alpha_{\mathrm{c}}$ results from a balance between in-plane stresses in the plate and the energy needed to advance the crack: it can in fact be computed from the mechanical and fracture properties of the film. We refer to Ref. 8] for a more detailed presentation of this geometrical fracture model and its rational derivation from elementary principles of fracture and plate mechanics.

\section{Experimental and numerical results}

A numerical simulation of this geometrical model yields oscillatory crack paths over a wide range of values of the parameters $\alpha_{c}$ and $\beta$. In Fig. 1 we compare typical computed (right) and experimental (left) crack paths. The non-sinusoidal crack patterns are in remarkably good agreement. Their wavelength and amplitude depend weakly on the parameters $\alpha_{\mathrm{c}}$ and $\beta$ of the model and their overall shape is almost independent of them.

The sequential processes of the crack propagation over a single period is presented and discussed in Figure 3 for both the experiments and numerics. The numerical simulations were run with parameters $\alpha=.153$ and $\beta=\pi / 2$. The evolution of the crack paths demonstrates strong similarities between the experiments and the simulation based on our model.

Our geometrical model includes a single length scale, the size of the cutting tip, and does not take into account its driving speed. In fact, we have performed experiments for $0.06 \mathrm{~mm} / \mathrm{s}<v<64 \mathrm{~mm} / \mathrm{s}$ and found no influence of speed of the patterns. We have also checked experimentally, for a fixed tip size $w=2 \mathrm{~mm}$, the pattern to be independent of the sheet's width, $D$, within the range $3.3 w<D<21 w$, in agreement with the predictions of the model. This feature is a priori surprising since one could have thought of $D$ as one of the natural length scales. Having determined that there is only one length scale $w$ in the problem, one expects both the wavelength and amplitude of the oscillations to scale linearly with it. Indeed, as shown on Fig. 4 we experimentally find a linear dependence of the amplitude and wavelength on the tip width over almost three decades, for a variety of polymeric materials and thicknesses. This supports a purely geometric fracture mechanism in this regime. Note that this linear behaviour breaks down in the vicinity of the transition region between straight and oscillatory paths: there, the sheet can no longer be considered thin relative to the cutting tip and the model's assumptions are not valid anymore (the plate bending and stretching energies become of the same order of magnitude).

To conclude, we have reported a novel mode of oscillatory crack propagation as a cutting tip of moderately large width is driven through a thin polymer sheet. We proposed a geometric model for these cracks, which retains the salient mechanical properties of thin elastic sheets: our scenario is based on the coupling between the direction of crack propagation and large deflections of the plate. Although very simple, such a model reproduces surprisingly many experimental features of the cracks, including their shape and dynamics. The crack path amplitude and wavelength were found independent of the driving speed, of the thickness and lateral width of the sheet, and scale linearly with the 

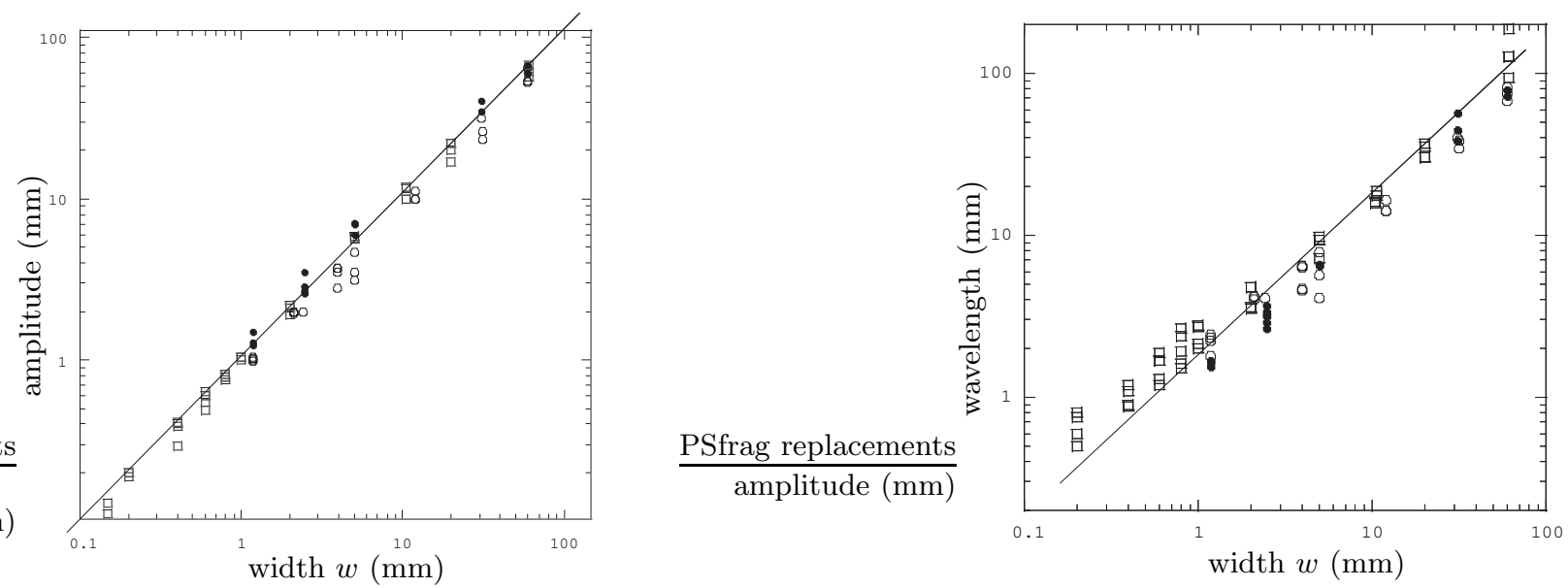

FIG. 4: Log-log plots of the peak-peak amplitude and wavelength of the oscillations as a function of tip width $w$ for various materials and thicknesses (polypropylene 25 to $53 \mu \mathrm{m}$ : open symbols, cellulose acetate 100 and $130 \mu \mathrm{m}$ : closed symbols), and for both cylinders $(\circ \bullet)$ and rectangular blades $(\square)$ : amplitude and wavelength organize on slope 1 lines, on a range of almost 3 decades. The straight lines represent the best linear fits with slope 1.

size of the object, in agreement with the model. This explains why this phenomenon is highly robust and insensitive to irregularities in the speed and boundary conditions, and can even be observed if the cutting is done by hand.

The authors wish to thank Mokhtar Adda Bedia for stimulating discussion, and Charles Baroud for putting in contact the Paris and Manchester groups who started working on this subject independently.

[1] Yuse A., Sano M. , Nature (London) 362 (1993) 329-331 .

[2] Ronsin O., Heslot F.and Perrin B., experimental study of quasistatic brittle crack propagation Phys. Rev. Let. 75 (1995)

[3] Adda-Bedia M. and Pomeau Y., Crack instabilities of a heated glass strip, Phys. Rev. E 52 (1995) 4105-4113.

[4] Yang B. \& Ravi-Chandar K., crack path instability in a quenched glass plate, J. Mech. Phys. Solids 49 (2001) 91-130

[5] Deegan R., Chheda S., Patel L., Marder M., Swinney H., Wavy and rough crack in Silicon, 2002 preprint

[6] Deegan R., Petersan P., Marder M., Swinney H., Oscillating fracture paths in rubber Phys. Rev. Let. 88 (2002)

[7] Pogorelov A., Bending of surfaces and stability of shells, Translation of mathematical monographs. AMS, 1988.

[8] B. Audoly, P. M. Reis, B. Roman, D. Vallet, V. Vigui, S. DeVilliers, in preparation. 